\title{
Numerical Simulation of Frost Formation on a Plate-Fin Evaporator
}

\author{
Ehsan Afrasiabian ${ }^{1}$, Oleg Iliev ${ }^{1}$, Stefano Lazzari $^{2}$, Carlo Isetti $^{3}$ \\ ${ }^{1}$ Fraunhofer-ITWM \\ Fraunhofer-Platz 1, 67663, Kaiserslautern, Germany \\ Ehsan.afrasiabian@itwm.fraunhofer.de; Oleg.iliev@itwm.fraunhofer.de \\ ${ }^{2}$ Department of Architecture and Design (DAD), University of Genoa \\ Stradone S. Agostino, 37 - 16123, Italy \\ stefano.lazzari@unige.it \\ ${ }^{3}$ TICASS scrl, Innovative Technologies for Environmental Control and Sustainable Development \\ Via B. Bosco, 57/4 Genoa, Italy \\ isetti@leonardo.arch.unige.it
}

\begin{abstract}
In the present paper, the Eulerian-granular model is adopted, to predict the frost growth on one channel of a plate-fin evaporator. A proper mass transfer model and modified frosting criteria are used to simulate the frost formation process. First, the model is validated with experimental data obtained under various operating conditions. The numerical predictions for the frost thickness and density are in good agreement with available experimental data. Furthermore, a parametric analysis is carried out to study the impact of the geometrical parameters of a three-dimensional plate-fin evaporator. A qualitative comparison shows a good agreement between the numerical data and experimental observations reported in the literature. One interesting outcome emerging from this study is that the distance between refrigerant tubes can play an important role in the frosting time.
\end{abstract}

Keywords: Frost formation, Evaporator, Heat pump, CFD, Heat and mass transfer, Densification.

\section{Introduction}

Recently there has been growing interest in using heat pumps for various industrial, commercial and residential applications, especially in the air-conditioning and automotive fields [1]. However, forming frost layers on air-cooled heat exchangers is a major issue that could jeopardize the high efficiency and performance of such devices. In fact, frost starts forming when humid air faces a surface having temperature lower than both zero Celsius degrees and the dew point for the considered air humidity ratio. The formed layer adds further thermal resistance to the system and reduces the transferred heat from the air to the refrigerant through the heat exchanger. Moreover, by increasing the thickness of the frost layer, the airflow through the heat exchanger decreases and convective heat transfer drops as well. Consequently, the heat capacity of the heat exchanger will reduce drastically. If the device keeps working, the energy consumption will rise and the system could fail finally. Thus, to avoid such a problem and protect the system from losing its efficiency, defrosting cycle should start to remove the formed frost from the surface. Although many researchers have studied frosting and defrosting processes, further investigations are required to gain a better insight into an effective defrosting treatment.

Frost formation is a complicated phenomenon involving simultaneous heat and mass transfer. In frosting process, the super-saturation degree of water vapour plays a key role as the driving force for water molecules to overcome Gibbs free energy and form ice nuclei on the surface [2]. After forming initial ice nuclei, ice crystals propagate to cover the surface and form a layer of frost [3]. From this stage, frost is considered as a porous structure consisting of ice crystals and air gaps [4]. In addition to deposition process causing the frost layer growth, water vapour molecules diffuse from the airside into the frost layer that leads to an increase in frost density [2]. In the last few decades, several models have been proposed to study frost formation process, mainly on a flat plate. In this light, some models just consider the frost side by replacing the airside effects with empirical correlations [5], while others take into account both airflow and frost layer simultaneously [6]. Armengol et al. [7] developed a two-dimensional model for frost formation with an interface condition to couple two domains. Cui et al. $[8,9]$ applied the nucleation theorem to model the mass transfer for frost formation process by using a two-fluid approach for both phases. They used an Eulerian-Eulerian model for a multiphase and multi-component system. 
Later, Kim et al. [10] established a Computational Fluid Dynamics (CFD) model in which mass transfer occurs due to the gradient of the water vapour concentration at frost/air interface. Recently, Wu et al. [11] applied a new model for the phase change mass transfer to simulate the frost formation on a cold plate and later, on a fin-and-tube heat exchanger [12]. In this model, the driving force for mass transfer is due to the super-saturation degree of water vapour and contributes to the frost thickening and densification processes.

In this study, an Eulerian-granular multiphase model is applied to simulate the interaction between the frost layer and humid air by solving the same governing equations for each phase. The mass transfer model proposed by Wu et al. [13] is implemented via User-Defined Functions (UDFs) in ANSYS-FLUENT package to simulate the frost layer growth and densification processes. Moreover, the frosting criterion is modified by separating velocity and super-saturation conditions. First, the model is validated for a range of experimental data and then frost formation on a three-dimensional geometry of a plate-fin evaporator is modelled to carry out a parametric analysis.

\section{Numerical Model}

The Eulerian-granular multiphase model, from ANSYS-FLUENT package, is used in this study. This model is a twofluid approach in which both primary and secondary phases coexist in each cell of the computational domain. The fraction of volume $(\alpha)$, occupied by each phase, is governed by Eq. (1), where $i$ and $a$ stand for the ice and humid air phases respectively.

$$
\alpha_{i}+\alpha_{a}=1
$$

In this approach, both phases are treated as continuous phases mixing and interacting with each other, while the size of the dispersed phase is reflected in a drag function. Two sets of effective conservation equations for mass, momentum, and energy are solved by sharing the same pressure field, whilst they are coupled via the interface exchange coefficients of momentum and energy as follows:

$$
\begin{aligned}
\frac{\partial}{\partial t}\left(\alpha_{i} \rho_{i}\right)+\nabla \cdot\left(\alpha_{i} \rho_{i} \vec{u}_{i}\right) & =S_{m i}, \quad S_{m i}=\dot{m}_{a i} \\
\frac{\partial}{\partial t}\left(\alpha_{a} \rho_{a}\right)+\nabla \cdot\left(\alpha_{a} \rho_{a} \vec{u}_{a}\right) & =S_{m a},
\end{aligned}
$$

$$
\begin{aligned}
& \frac{\partial}{\partial t}\left(\alpha_{i} \rho_{i} \vec{u}_{i}\right)+\nabla \cdot\left(\alpha_{i} \rho_{i} \vec{u}_{i} \vec{u}_{i}\right)=K_{a i}\left(\vec{u}_{a}-\vec{u}_{i}\right)-\alpha_{i} \nabla p+\nabla \cdot \overline{\bar{\tau}}_{i}+\alpha_{i} \rho_{i} \vec{g}+S_{u i}, \quad S_{u i}=\dot{m}_{a i} \vec{u}_{i} \\
& \frac{\partial}{\partial t}\left(\alpha_{a} \rho_{a} \vec{u}_{a}\right)+\nabla \cdot\left(\alpha_{a} \rho_{a} \vec{u}_{a} \vec{u}_{a}\right)=K_{i a}\left(\vec{u}_{i}-\vec{u}_{a}\right)-\alpha_{a} \nabla p+\nabla \cdot \overline{\bar{\tau}}_{a}+\alpha_{a} \rho_{a} \vec{g}+S_{u a}, \quad S_{u a}=-\dot{m}_{a i} \vec{u}_{a}
\end{aligned}
$$

$$
\begin{aligned}
& \frac{\partial}{\partial t}\left(\alpha_{i} \rho_{i} h_{i}\right)+\nabla \cdot\left(\alpha_{i} \rho_{i} \vec{u}_{i} h_{i}\right)=Q_{a i}-\alpha_{i} \frac{\partial p_{i}}{\partial t}+\overline{\bar{\tau}}_{i}: \nabla \vec{u}_{i}-\nabla \cdot \vec{q}_{i}+S_{h i}, \quad S_{h i}=\dot{m}_{a i}\left(h_{v}+l_{v}\right) \\
& \frac{\partial}{\partial t}\left(\alpha_{a} \rho_{a} h_{a}\right)+\nabla \cdot\left(\alpha_{a} \rho_{a} \vec{u}_{a} h_{a}\right)=Q_{i a}-\alpha_{a} \frac{\partial p_{a}}{\partial t}+\overline{\bar{\tau}}_{a}: \nabla \vec{u}_{a}-\nabla \cdot \vec{q}_{a}+S_{h a}, \quad S_{h a}=-\dot{m}_{a i} h_{v}
\end{aligned}
$$

Here $K$ and $Q$ are interface exchange coefficients for the momentum and the intensity of heat between the two phases involved. In the above governing equations, $\rho$ is the density, $p$ is the pressure, $\vec{u}$ represents the velocity vector, $\vec{q}$ is the heat flux, $h$ is the specific enthalpy, and $\overline{\bar{\tau}}$ is the stress-strain tensor of air or ice. Furthermore, the water vapour mass fraction $\left(w_{v}\right)$ is governed by the following transport equation: 


$$
\frac{\partial}{\partial t}\left(\alpha_{a} \rho_{a} w_{v}\right)+\nabla \cdot\left(\alpha_{a} \rho_{a} w_{v} \vec{u}_{a}\right)=\nabla \cdot\left(\rho_{a} D_{H_{2} o} \nabla w_{v}\right)+S_{v a}, \quad S_{v a}=-\dot{m}_{a i}
$$

Here $D_{\mathrm{H}_{2} \mathrm{O}}$ is the diffusivity of water vapour in the dry air and since the system is dilute, a constant dilute approximation for diffusivity of water vapour in the air is used. Moreover, in the source terms $(S)$ in Eqs. $(2-5), l_{v}$ is the released latent heat per unit mass.

In this approach, Wen-Yu's model is used and the fluid-solid momentum exchange coefficient is given as [14]:

$$
\begin{gathered}
K=\frac{3}{4} C_{D} \frac{\alpha_{i} \alpha_{a}\left|\overrightarrow{u_{a}}-\overrightarrow{u_{\imath}}\right|}{d_{i}} \alpha_{a}^{-2.65} \\
C_{D}=\frac{24}{\alpha_{a} R e_{i}}\left[1+0.15\left(\alpha_{a} R e_{i}\right)^{0.687}\right] \\
R e_{i}=\frac{\rho_{a} d_{i}\left|\overrightarrow{u_{l}}-\overrightarrow{u_{a}}\right|}{\mu_{a}}
\end{gathered}
$$

In Eqs. (6-8), $C_{D}$ is the drag function, $d_{i}$ is the ice particles diameter, and $R e_{i}$ is the relative Reynolds number. Another important relationship between ice and humid airflow gives the exchanged heat flux per unit area, as stated by Eq.(9).

$$
\begin{gathered}
Q_{i a}=C_{h}\left(T_{i}-T_{a}\right) \\
C_{h}=\frac{6 \lambda_{a} \alpha_{a} \alpha_{i} N u_{i a}}{d_{i}^{2}} \\
N u_{i}=\left(7-10 \alpha_{a}+5 \alpha_{a}^{2}\right)\left(1+0.7 \operatorname{Re}_{i}^{0.2} \operatorname{Pr}^{\frac{1}{3}}\right)+\left(1.33-2.4 \alpha_{a}+a .2 \alpha_{a}^{2}\right) \operatorname{Re}_{i}^{0.7} \operatorname{Pr}^{\frac{1}{3}}
\end{gathered}
$$

where $C_{h}$ is the heat transfer coefficient between the two phases and given by Eq. (10). Here $\lambda_{a}$ is the thermal conductivity of the humid air, $P r$ is the Prandtl number, and the Nusselt number $N u_{i a}$ is given by Gunn model [14].

In source terms used in Eqs. (2-5), $\dot{m}_{a i}$ is the mass transfer rate to the ice phase and is defined as [13]:

$$
\begin{gathered}
\dot{m}_{a i}=\gamma \alpha_{a} \rho_{a} w_{v} \frac{w_{v}-w_{v s}}{w_{v s}} \\
w_{v s}=0.622 \frac{p_{v s}}{p_{0}-p_{v s}}
\end{gathered}
$$

where $\gamma$ is a time relaxation coefficient, $w_{v s}$ is the saturated water vapour mass fraction at temperature $T$, and $p_{0}$ is the atmospheric pressure. Eq. (13) gives the saturated water vapour pressure for temperature $T[15]$ : 


$$
p_{v S}= \begin{cases}0.61121 \exp \left(\left(18.678-\frac{T}{234.5}\right)\left(\frac{T}{257.14+T}\right)\right), & T \geq 0^{\circ} \mathrm{C} \\ 0.61115 \exp \left(\left(23.036-\frac{T}{333.7}\right)\left(\frac{T}{279.82+T}\right)\right), & T<0^{\circ} \mathrm{C}\end{cases}
$$

According to the mass transfer criterion established in [13], a non-dimensional coefficient B, phase change driving force, and humid air velocity are used to describe the frost growth and densification processes. In the cited work, the frost formation criterion is assumed to be $\left(w_{v}-w_{v s}\right) / w_{v s} \geq B \cdot \vec{u}_{a} / \vec{u}_{i n}$ but since the velocity and water vapour concentration are two independent physical parameters, it seems that by separating the frosting condition, numerical results are less sensitive to the parameter B and are in better agreement with experimental data. Thus, the mass transfer rate contributing to both frost thickening and densification would be as:

$$
\dot{m}_{a i}=\gamma \alpha_{a} \rho_{a} w_{v} \frac{w_{v}-w_{v s}}{w_{v s}} \quad \text { if } w_{v}-w_{v s}>0 \text { and } \frac{\vec{u}_{a}}{\vec{u}_{i n}} \leq B
$$

In fact, since the Gibbs free energy has the maximum value for the homogenous nucleation far from the solid surfaces and super-saturation degree is always maximum at cold surfaces (i.e. frost/air interface), heterogeneous nucleation is the favoured mechanism for frosting happening inside the frost layer or at a solid surface [3]. It means that if the velocity is low enough (inside and at the surface of the frost layer) and the humid air is supersaturated, the mass transfer happens which is clearly reflected by the frosting condition in Eq. (15).

\section{Solution Scheme}

The Species and Eulerian multiphase models of the ANSYS - FLUENT package were applied to simulate the humid air as the primary phase, while the secondary phase was considered ice droplets with the diameter of $10^{-5} \mathrm{~m}$. All physical properties of the humid air are weighted averages of the dry air and water vapour properties. Governing equations were discretized using the QUICK method, while pressure and velocity fields were coupled via the Coupled algorithm. Due to the low speed of the frosting process, the time step was set to $1 \mathrm{~s}$ and results were examined for shorter time steps with no remarkable differences.

\section{Results and Discussion}

\subsection{Validation}

Verification and validation of simulation models were conducted by comparing numerical results with experimental data reported by Hermes et al. [16]. First, the Eulerian multiphase model was applied to simulate the frost formation on a cold flat plate with the temperatures ranging from -16 to $-4{ }^{\circ} \mathrm{C}$ with the same conditions listed in Table (1). As Figure (1) shows, predictions of the numerical model (in red) are in agreement with experimental data (in black). Figure (1) reveals that the average frost thickness $(\delta)$ increases as the temperature of the cold plate decreases, which is consistent with a number of previous reports.

Table 1: Frosting conditions.

\begin{tabular}{|l|l|l|l|l|}
\hline Case & $\boldsymbol{T}_{\text {wall }}\left(\boldsymbol{C}^{\circ}\right)$ & $\boldsymbol{T}_{\boldsymbol{a}_{\text {in }}}\left(\boldsymbol{C}^{\circ}\right)$ & $\boldsymbol{u}_{\text {in }}(\boldsymbol{m} / \mathbf{s})$ & $\boldsymbol{R H}(\%)$ \\
\hline Hermes et al. (2009) [16] & $-16 \sim-4$ & 16 & 0.7 & 85 \\
\hline Sommers et al. (2016) [17] & -7 & 22 & 0.7 & 80 \\
\hline Plate-fin evaporator & -8 & 1.7 & - & 85 \\
\hline
\end{tabular}

Furthermore, the numerical model was validated by simulating the experiments conducted by Sommers et al. [17]. They analysed the frost formation on surfaces with the same size $(99.5 \mathrm{~mm} \times 80.2 \mathrm{~mm} \times 3.4 \mathrm{~mm})$ and different 
wettability. As indicated in Table (2), the inlet air velocity was set to $0.7 \mathrm{~m} / \mathrm{s}$, relative humidity (RH) was kept at $85 \%$, and the surface temperature was $-7^{\circ} \mathrm{C}$. As Figures (2 and 3) show, and considering reported uncertainties in the experiments, numerical predictions agree well with experimental data with the error less than 7\% and 5\% (after 30 min) for the average frost thickness and density $\left(\rho_{f}\right)$ respectively.

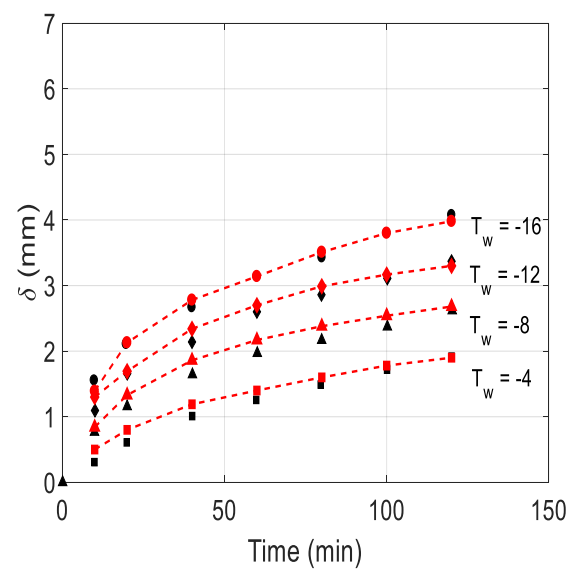

Fig. 1: Averaged frost thickness; current work vs. experimental data [16].

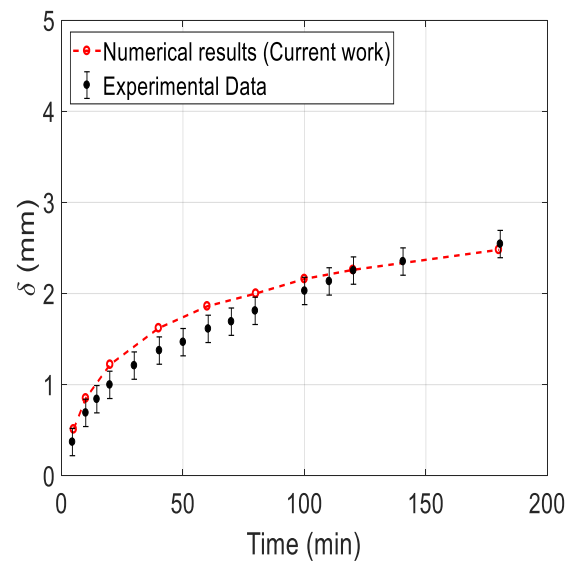

Fig. 2: Averaged frost thickness; current work vs. experimental data [17].

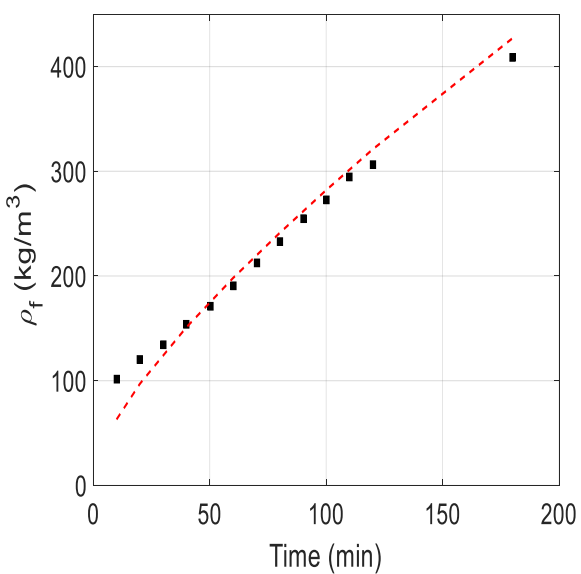

Fig. 3: Averaged frost density; current work vs. experimental data [17]. 


\subsection{Frosting on a Plate-Fin Evaporator}

Frost growth on an evaporator surface or a micro-channel heat exchanger, when used in outdoor heat pump or air conditioning systems, can be analysed using the current model. As illustrated in Figure (4) one section of a plate-fin evaporator was modelled in a three-dimensional computational domain and conditions were set as listed in Table (2) to analyse the local frost thickness and distribution of the frost density.

As shown in the bottom left -Figure (4), where the computational domain is illustrated, a cooling block with size of $\mathrm{H} \times \mathrm{X} \times 24 \mathrm{~mm}$, is extended upwind with an insulated (non-cooling) block. Similar approach is used often in CFD, including simulations of frost formation. The existence of inlet development area, which is free from frost formation, leads to better convergence. In the non-cooling block, there is no fin and all the boundary conditions are symmetry except for the inlet in which $\alpha_{i}=0, p_{\text {total }}=p_{i n}, T_{a}=T_{a_{i n}}, w_{v}=w_{i n}$. Moreover, at the outlet in the cooling block $p_{\text {static }}=101 \mathrm{kPa}$. Furthermore, the fins, upper, and bottom surfaces of the channel, in the cooling block, are walls with the no-slip velocity boundary condition and $\vec{u}_{a}=\vec{u}_{i}=0, T=T_{w a l l},\left(\partial w_{v}\right) / \partial n=0,\left(\partial \alpha_{i}\right) / \partial n=0$ where $n$ is the surface normal vector.

In this work, a parametric analysis was carried out in order to gain insights into the influence of geometrical features on the frost properties. Three different cases were studied with the geometrical parameters listed in Table (2). The first case was considered the reference geometry, as illustrated in Figure (4), while the other cases varied in the height $(\mathrm{H})$ or bottom width (X2) of the channel and the top width (X1) was fixed. In order to compare the numerical results of different cases, the following non-dimensional parameters and variables are introduced:

$$
\begin{gathered}
A_{\text {ref }}=A_{1}, \quad p_{i}^{*}=\frac{p_{i}-p_{0}}{p_{1}-p_{0}}, \quad m_{f i}^{*}=\frac{m_{f i}}{m_{f_{\text {ref }}}}, \quad u_{i}^{*}=\frac{u_{i}}{u_{\text {ref }}}, \quad i=1,2,3 \\
m_{f_{\text {ref }}}=m_{f 1\left(t_{\text {ref }}\right)}
\end{gathered}
$$

where $A$ and $m$ are face area and accumulated frost mass respectively.

As Figure (4) shows, at the beginning frost starts forming on the surface due to the deposition of water vapour and as time goes on, the thickness of the layer increases, leading to the airflow blockage. On the other hand, due to the diffusion of water vapour molecules, the density of frost layer increases continuously. From a qualitative point of view there is a good agreement between the numerical results and experimental observations [18]; however, there is no quantitative comparison. In the second case, the FPI (Fins Per Inch) increased from 10.6 to12.7 while the face area $(A)$ of the section was constant. In this case, the non-dimensional pressure drop $\left(p^{*}\right)$ imposed on the boundaries of the computational domain, was 1.3 to have the same initial mass flow rate of the humid air as the reference case. In the third case, the face area decreased to $72.7 \%$ of the reference area by reducing X2 and increasing the fin density per unit length. In this situation, the total number of the fins per unit are increased and $p^{*}$ reached 1.6 to have the same initial inlet airflow velocity as the reference value, leading to a lower airflow rate through the channel.

Figure (5) compares the airflow velocity reduction and frost mass accumulation $\left(\mathrm{m}^{*}\right)$ on the channel for different cases. The reference time $\left(t_{r e f}\right)$ is when, in the reference case, the velocity drops to $23 \%$ of its initial value $\left(u_{\text {init }}=\right.$ $1.5 \mathrm{~m} / \mathrm{s}$ ). As shown in Figure (5), the black line (case No. 3) reports less velocity reduction and frost mass (in one section) than the blue line (case Ref.). It means that for the reference case frost grows faster and the airflow blockage is significantly much more for a given time. This phenomenon could be due to the less mass flow rate through a single channel that carries less water vapour in case No. 3. Furthermore, as mentioned before, $p^{*}$ is 1.6 times bigger, for the case No. 3 compared to the reference case. It means that more power for the fan is needed to provide the same velocity at the inlet. 

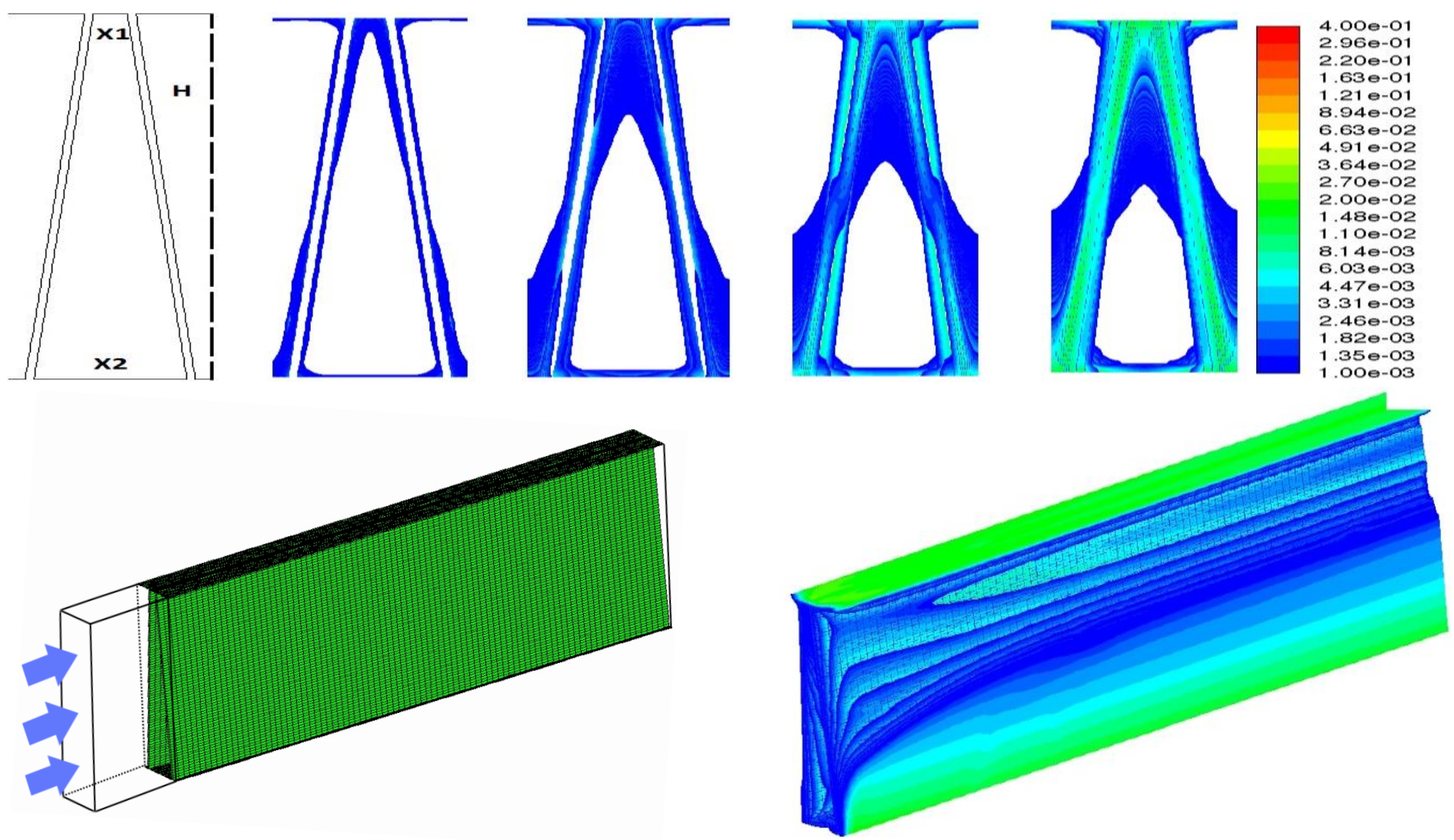

Fig. 4: Time-wise variation of $\alpha_{i}$, top) front view, bottom) isometric view (Computational domain on the left).

Table 2: Geometrical parameters.

\begin{tabular}{|l|c|c|c|c|c|c|}
\hline Sample No. & Fins per inch $(\boldsymbol{F P I})$ & $\begin{array}{c}\mathbf{X 1} \\
(\mathbf{m m})\end{array}$ & $\begin{array}{c}\mathbf{X} \mathbf{2} \\
(\mathbf{m m})\end{array}$ & $\begin{array}{c}\boldsymbol{H} \\
(\mathbf{m m})\end{array}$ & $\begin{array}{c}\text { Fin thickness } \\
(\mathbf{m m})\end{array}$ & $\begin{array}{c}\text { Face area } \\
\left(\mathbf{1 0}^{-\mathbf{6}} \mathbf{m}^{\mathbf{2}}\right)\end{array}$ \\
\hline 1 (Ref.) & 10.6 & 0.4 & 1.8 & 8.0 & 0.1 & 4.4 \\
\hline 2 & 12.7 & 0.4 & 1.4 & 9.8 & 0.1 & 4.4 \\
\hline 3 & 14.1 & 0.4 & 1.2 & 8.0 & 0.1 & 3.2 \\
\hline
\end{tabular}

Another interesting outcome of the current study emerges from the simulation of case No. 2. As Figure (5) shows, the frost growth rate for higher FPI (case No. 2) is lower than the reference case with the same face area while the accumulated frost mass on the surface is slightly lower. In other words, as Figure (5) displays, by decreasing the fin's bottom width (X2) and increasing the fin's height $(\mathrm{H})$ the frosting time is significantly prolonged. In accordance with the present results, a previous experimental study for louvered fin evaporator [18] has demonstrated that in some circumstances the increase in height could result in a longer frosting time. In this way and compared to the reference case, case No. 2 would be a better choice with higher coefficient of performance when the evaporator faces the undesirable frosting phenomenon. 


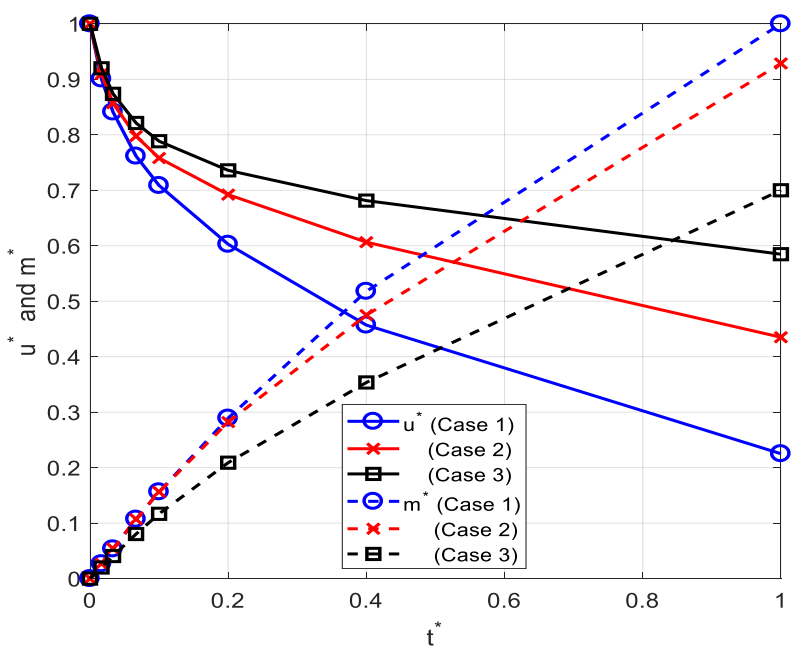

Fig. 5: Frost mass and airflow velocity vs. time.

\section{Conclusion}

In the present work, a numerical study was performed to predict frost growth on one channel of a plate-fin evaporator. The numerical approach proposed by [13] was used while the frosting criteria were modified by separating the velocity and super-saturation conditions. First, the model was validated by comparing the numerical results with experimental data obtained under various operating conditions for the frost thickness and density. These results agreed well with available experimental data. Then, the Eulerian-granular multiphase model was used to simulate the frost formation growth on a three-dimensional plate-fin evaporator and the numerical results were in keeping with previous experimental observations. Finally, a parametric analysis was carried out and different geometries were investigated. One interesting outcome emerging from this study was that the frosting rate not only is affected by the FPI parameter but also the distance between refrigerant tubes can play an important role in the frosting time.

\section{Acknowledgement}

This work is part of XERIC project that has received funding from the European Union's Horizon 2020 research and innovation programme under grant agreement $\mathrm{N}^{\circ} 653605$.

\section{References}

[1] K. J. Chua, S. Chou and W. M. Yang, "Advances in heat pump systems: A review," Applied Energy, vol. 87, no. 12, pp. 3611-3624, 2010.

[2] B. Na and R. L. Webb, "A fundamental understanding of factors affecting frost nucleation.," International Journal of Heat and Mass Transfer, vol. 46, no. 20, pp. 3797-3808, 2003.

[3] S. Nath and J. B. Boreyko, "On localized vapor pressure gradients governing condensation and frost phenomena," Langmuir, vol. 32, no. 33, pp. 8350-8365, 2016.

[4] Y. Hayashi, A. Aoki, S. Adaohi and K. Hori, "Study of frost properties correlating with frost formation types," Journal of heat transfer, vol. 99, no. 239, 1977.

[5] Y. Tao, R. Besant and K. Rezkallah, "A mathematical model for predicting the densification and growth of frost on a flat plate," Int. J. Heat Mass Transfer, vol. 36, pp. 353-363, 1993.

[6] K. Lee, S. Jhee and D.-K. Yang, "Prediction of the frost formation on a cold flat surface," Int. J. Heat Mass Transfer, vol. 46, pp. 3789-3796, 2003.

[7] J. M. Armengol, C. T. Salinas, J. Xaman and K. A. R. Ismail, "Modeling of frost formation over parallel cold plates considering a two-dimensional growth rate," International Journal of Thermal Sciences , vol. 104, pp. 245-256, 2016.

[8] J. Cui, W. Z. Li, Y. Liu and Z. Y. Jiang, "A new time-and space-dependent model for predicting frost formation," 
Applied Thermal Engineering, vol. 31, no. 4, pp. 447-457, 2011.

[9] J. Cui, W. Z. Li, Y. Liu and Y. S. Zhao, "A new model for predicting performance of fin-and-tube heat exchanger under frost condition," International Journal of Heat and Fluid Flow, vol. 32, no. 1, pp. 249-260, 2011.

[10] D. Kim, C. Kim and K.-S. Lee., "Frosting model for predicting macroscopic and local frost behaviors on a cold plate," International Journal of Heat and Mass Transfer, vol. 82, pp. 135-142, 2015.

[11] X. M. Wu, Q. Ma, F. Chu and S. Hu, "Phase change mass transfer model for frost growth and densification," International Journal of Heat and Mass Transfer, vol. 96, pp. 11-19, 2016.

[12] X. Wu, Q. Ma and F. Chu, "Numerical Simulation of Frosting on Fin-and-Tube Heat Exchanger Surfaces," Journal of Thermal Science and Engineering Applications, vol. 9, no. 3, p. 031007, 2017.

[13] X. Wu, F. Chu and Q. Ma, "Frosting model based on phase change driving force," International Journal of Heat and Mass Transfer, vol. 110, p. 760-767, 2017.

[14] Fluent, A. N. S. Y. S, Release 15.0, Theory Guide, 2013.

[15] Buck Research CR-1A User's Manual, Appendix 1, 1996.

[16] C. J. Hermes, R. O. Piucco, J. R. B. Jr. and C. Melo, "A study of frost growth and densification on flat surfaces," Experimental Thermal and Fluid Science, vol. 33, p. 371-379, 2009.

[17] A. D. Sommers, N. L. Truster, A. C. Napora, A. C. Riechman and E. J. Caraballo, "Densification of frost on hydrophilic and hydrophobic substrates - Examining the effect of surface wettability," Experimental Thermal and Fluid Science, vol. 75, p. 25-34, 2016.

[18] E. Moallem, T. Hong, L. Cremaschi and D. E. Fisher, "Experimental investigation of adverse effect of frost formation on microchannel evaporators, part 1: Effect of fin geometry and environmental effects," International Journal of Refrigeration, vol. 36, no. 6, pp. 1762-1775, 2013. 